\title{
ANÁLISE DA EFICIÊNCIA DOS FUNDOS DE INVESTIMENTOS IMOBILIÁRIOS
}

\author{
EFFICIENCY ANALYSIS OF REAL ESTATE INVESTIMENT FUNDS
}

DOI: http://dx.doi.org/10.12712/rpca.v5i1.21

Jorge Mizuno

Pontifícia Universidade Católica do Paraná

\section{Luis Carlos Duclós}

Pontifícia Universidade Católica do Paraná

Wesley Vieira da Silva

Pontifícia Universidade Católica do Paraná

\section{Daniela Torres da Rocha}

Pontifícia Universidade Católica do Paraná

\section{RESUMO}

Este artigo tem o objetivo de analisar eficiência do uso dos recursos dos Fundos de Investimentos Imobiliários utilizando a métrica $D E A$ com aderência à teoria Visão Baseada em Recursos. A métrica $D E A$ é uma aplicação da técnica de programação linear utilizada para medir a eficiência relativa de uma determinada unidade operativa em relação às demais unidades com alguns objetivos definidos. Os resultados deste estudo sugerem os Fundos são mais eficientes no uso de recursos do Patrimônio Líquido e Ativo Total do que o uso de Receitas. Verificou-se também, que a teoria Visão Baseada em Recursos apresentou aderência aos aspectos que explicam as razões de forma abrangente, da ocorrência de grandes diferenças de desempenho dada a existência de vantagem competitiva nos Fundos de Investimentos Imobiliários analisados.

Palavras-chave: Visão baseada em recursos. Fundos de investimentos imobiliários. DEA.

\begin{abstract}
This article aims to analyze the efficiency of resource use of property investiment funds using the metric DEA with adherence to the Resource Based View theory. The metric is an application of DEA linear programming technique used to measure the relative efficiency of a particular operating unit in relation to other units with some defined goals. The results of this study suggest the Funds are more efficient in resource use Equity and Total Assets of the use of revenue. It was also noted that the Resource Based View theory presented adherence to those aspects which explained comprehensively, the occurrence of large differences in performance due to the existence of competitive advantage in property investment funds analyzed.
\end{abstract}

Keywords: Resource based view. Real estate investment funds. DEA. 



\section{INTRODUÇÃO}

Os Fundos de Investimentos Imobiliários FII representam uma forma de captação de recursos públicos por meio de cotas representativas de valores mobiliários de renda variável. Foi regulado pela Lei 8668/93, modificada pela Lei 9779/99, numa adaptação do instituto do "Trust" do direito comum anglo-saxão ao Direito Brasileiro. Composto de grupo de investidores gerido por instituição financeira sob fiscalização da Comissão de Valores Mobiliários - CVM de acordo com as Instruções 205/94 e 206/94.

Com o bom momento de negócios vivenciado pela construção civil, os setores que formam as fontes de recursos financeiros de longo prazo se estruturam para aproveitar as boas oportunidades de investimentos. Neste contexto, os Fundos de Investimentos Imobiliários FII dado o seu formato adequado de associar riscos e retornos no longo prazo, participam com vantagens quando comparado com outros participantes deste mercado.

No Brasil, os 72 FII registrados na CVM somam em patrimônio líquido $\mathrm{R} \$ 3,48$ bilhões em 31 de Maio de 2008, operando em Shopping Center, Hospitais, Hotéis, Complexos Industriais, e Empreendimentos Comerciais e Residenciais. Em 2003, esse valor era R 2,02 bilhões e nos Estados Unidos era de US\$ 6,35 bilhões.

Os fundamentos teóricos estabelecidos pela Visão Baseada em Recursos considerada neste trabalho apresentam forte aderência aos aspectos que explicam as razões de forma abrangente, da ocorrência de grandes diferenças de desempenho dada a existência de vantagem competitiva nos Fundos de Investimentos Imobiliários analisados. Além da heterogeneidade dos recursos utilizados pelos fundos, há os efeitos decorrentes das idiossincrasias reconhecidas por Rumelt (1991) e McGahan (1997) como importantes na explicação das diferenças dos resultados das empresas de um mesmo setor.

A métrica DEA é uma ferramenta que pode ser incorporada na rotina de análise de performance dos Fundos de Investimentos Imobiliários. É um método que utiliza modelagem do problema de programação linear exigindo pouca preparação e padronização dos dados e demonstrando flexibilidade para realizar simulações de resultados.

O objetivo desta pesquisa é analisar eficiência do uso dos recursos dos Fundos de Investimentos Imobiliários utilizando a métrica $D E A$ com aderência à teoria Visão Baseadaem Recursos.

O trabalho está estruturado em cinco seções que podem ser descritas da seguinte forma: a primeira refere-se a parte introdutória; a segunda diz respeito ao referencial teóricoempírico, onde são apresentados os principais conceitos acerca da Visão Baseada em Recursos e dos Fundos de Investimentos Imobiliários; a terceira trata da metodologia utilizada na pesquisa; a quarta refere-se a apresentação e análise dos dados e a quinta traz as considerações finais e recomendações para elaboração de trabalhos futuros.

\section{REFERENCIAL TEÓRICO-EMPÍRICO}

\section{A VISÃO BASEADA EM RECURSOS (RBV)}

Entender as fontes de vantagem competitiva para empresas tem se tornado a maior área de pesquisa no campo de gerenciamento estratégico (Porter, 1985).

Para melhor avaliar as diferenças de performance é utilizada a abordagem baseada em recursos RBV a qual considera que a empresa é formada pelo conjunto de recursos, competências, processos e estrutura que, constituindo um ativo base, interagem no processo global de produção de bens e serviços.

A abordagem da estratégia baseada em recursos representa uma condição aplicável à empresas "como a construção de uma vantagem competitiva durável sobre um mercado específico, a partir da maneira 
como se cria valor pela configuração (produto, região, estruturas) e a coordenação dessas atividades sobre os diferentes mercados"(Brilman, 2001).

Segundo Porter (1996), eficácia operacional significa desempenhar atividades similares melhor do que os rivais. Refere-se a qualquer número de práticas que permitam a uma empresa utilizar melhor seus recursos.

As diferenças em eficácia operacional entre as empresas concorrentes são muitas. A forma de gerenciar seus funcionários, de aproveitar melhor os recursos, as escolhas por tecnologias, determinam ao final as diferenças na lucratividade entre os concorrentes decorrentes das variações de seus custos operacionais.

Segundo a teoria da RBV, o desempenho superior é sustentável quando uma organização tem recursos capazes de lhe render resultados extraordinários, e estes são raros (não disponíveis com facilidade para outras organizações), difíceis ou custosos de serem imitados, e a firma possui condições organizacionais para explorar as rendas geradas por tais recursos.

Barney (1991) define recursos como:

todos os ativos, capacidades, processos organizacionais, atributos da empresa, informações, conhecimentos etc., controlados pela empresa e que lhe permitem compreender e colocar em ação as estratégias susceptíveis de aumentar sua eficácia e sua eficiência.

Johnson (2007) divide os recursos em duas classes (tangíveis e intangíveis) e quatro categorias, tal como encontram-se descritos a seguir:

- Recursos físicos: refere-se ao número de máquinas, imóveis ou capacidade de produção da organização. A natureza desses recursos, tais como: idade, condição,capacidade e localização de cada um, vai determinar sua utilidade;

- Recursos financeiros: refere-se ao capital, dinheiro, devedores e credores, e fornecedores de dinheiro (acionistas, bancos, etc.);

- Recursos humanos: incluem-se o número e o mix (por exemplo: perfil demográfico) de pessoas numa organização. 0 recurso intangível das habilidades e conhecimentos de cada um também tende a ser importante. Isso se aplica tanto aos funcionários como às outras pessoas na rede de uma organização. Nas economias baseadas em conhecimento, as pessoas genuinamente se tornam o ativo mais valioso;

- Capital intelectual: é um aspecto importante dos ativos intangíveis de uma organização. Inclui patentes, marcas, sistemas empresariais e bancos de dados de clientes. Não deve haver dúvidas de que esses recursos têm seu valor, pois quando as empresas são vendidas, parte do valor é representada pela "clientela". Numa economia baseada em conhecimento, o capital intelectual tende a ser um ativo importante para muitas organizações.

Possuir os recursos é importante, mas, igualmente importante é como a organização os emprega. A eficiência e a eficácia de recursos físicos, financeiros e recursos humanos em uma organização depende não somente de sua disponibilidade, mas da forma de como são gerenciados. A otimização do uso compartilhado de todos os recursos em todas as suas dimensões estabelece o seu nível de competência que determina a ocorrência de vantagem competitiva.

Segundo Barney (1991) uma empresa obtém vantagem competitiva quando desenvolve estratégia de criação de valor não sendo imitado simultaneamente por qualquer atual ou potencial competidor. A condição de preservar ao longo do tempo o potencial de operar com vantagem competitiva cria uma dimensão superior denominada vantagem competitiva sustentada. Para ter este potencial, o recurso da empresa necessita ter quatro atributos: 
- Recursos Valiosos: deve permitir explorar as oportunidades ou neutralizar as ameaças doambiente;

- Recursos Raros: o conceito indica uma condição privilegiada de posse de um recurso em relação às empresas concorrentes;

- Recursos Imperfeitamente Imitáveis: representa uma condição da preservação da vantagem competitiva dos atributos valor e raridade em função da sua exclusividade. A impossibilidade de sua imitação mantém as vantagens competitivas sustentáveis;

- Condições Históricas: a formação de cultura única e valiosa estruturada nos primeiros estágios da história da empresa produz uma condição que não é possível imitar.

A condição da preservação de vantagem competitiva passa por ações de gerenciamento dos recursos valiosos da organização, visando a sua superação e blindagem para evitar que ações dos concorrentes para que possam produzir surpresas anulando os seusatributos.

Em ambientes com condições hiper-competitivas o ciclo de vida das vantagens competitivas tendem a ser menores, obrigando o processo de inovação a ser realizado em ritmo crescente. Os clientes têm mais escolha e as chances de estabelecer vantagem competitiva sustentável baseada num conjunto de competências duráveis se torna menos provável. Dada as oportunidades de novas experiências o cliente aceita a troca de fornecedor o que gera a quebra do acordo de fidelidade. Nesse contexto, algumas empresas mais ágeis conseguem atingir vantagem competitiva sobre outras que escolheram $o$ caminho da manutenção do padrão vigente de atendimento do cliente.

Teece (1997) propõe o desenvolvimento de capacidades dinâmicas nas organizações em ambientes que mudam rapidamente. Capacidade dinâmica significa a habilidade de uma organização em desenvolver e mudar competências para atender ambiente com hipercompetitividade. Sua configuração pode assumir características formais e informais, visíveis e invisíveis.

Embora em condições competitivas mais estáveis seja possível criar vantagem competitiva, em ambientes com hiper-competitividade a vantagem competitiva tem que ser desenvolvida tendo como base os aspectos de mudança, inovação e assimilação.

O desenvolvimento de novos recursos que tenham potencial de ampliar o nível de vantagem competitiva representa, neste contexto, a manutenção de atuar no mercado em condições favoráveis num cenário mais longo. Como resultado adicional, o desenvolvimento de novos recursos atende o processo de substituição dos recursos que, por ação deliberada da concorrência tenha imitado ou mesmo superado.

Considerando as características dos recursos que geram vantagem competitiva, sua obtenção passa por um processo de desenvolvimento interno com eficiente sistema de qualidade controlada pela empresa. Vantagem competitiva não se compra, se produz.

\section{FUNDOS DE INVESTIMENTOS IMOBILIÁRIOS - FII}

O Fundo Imobiliário foi regulado pela Lei 8668/93, modificada pela Lei 9779/99, numa adaptação do instituto do "Trust" do direito comum anglo-saxão ao Direito Brasileiro. Composto de grupo de investidores gerido por instituição financeira sob fiscalização da Comissão de Valores Mobiliários CVM de acordo com as Instruções 205/94 e 206/94.

Os Fundos de Investimentos Imobiliários FII representam uma forma de captação de recursos por meio de cotas representativas de valores mobiliários de renda variável. Essas cotas são parcelas de valores mobiliários não resgatáveis, tendo que vender a terceiros para retomar o investimento. Essas cotas são emitidas para subscrição mediante ofertas públicas e comercializadas no mercado secundário. Os recursos são aplicados em projetos imobiliários em construção ou concluídos e o patrimônio é composto desses projetos sejam eles parcialmente ou comotodo. 
Os FII podem alocar seus recursos em investimentos coletivos de renda variável em Imóveis na forma de condomínio comum, condomínio de unidades autônomas, empresa de propósito específico, empresa de participações, e debêntures de participação.

Os FII têm a vantagem de realizar as operações de compra e venda de cotas, parcial ou total, no mercado secundário sem os procedimentos operacionais de transações imobiliárias. Além disso, o recurso pode ser aplicado em vários empreendimentos simultaneamente.

Com relação aos aspectos tributários, os FII estão isentos de impostos, inclusive de Imposto de Renda, exceto sobre aplicações financeiras decorrentes de disponibilidade de recursos. Os benefícios fiscais são regulados pela Lei 9779/99.

De acordo com a Comissão de Valores Mobiliários - CVM, os FII estão sujeitos às mesmas condições de risco do mercado financeiro como um todo. A rentabilidade do investidor é decorrente da operação de locação, compra e venda de imóveis lastreados por um ou mais imóveis. O investidor não tem nenhuma ascendência ou responsabilidade sobre a administração ou risco de inadimplência do imóvel.

No Brasil, os 72 FII registrados na CVM somam em patrimônio líquido R\$3,48 bilhões em 31 de Maio de 2008, operando em Shopping Center, Hospitais, Hotéis, Complexos Industriais, e Empreendimentos Comerciais e Residenciais. Em 2003, esse valor era de aproximadamente R $\$ 2,02$ bilhões e nos Estados Unidos era US\$ 6,35 bilhões.

De acordo com a CVM, os FII no Brasil tendem a ser uma opção aos investidores brasileiros e estrangeiros que queiram realizar investimentos em aplicações imobiliárias, além de oferecer modalidades interessantes de operações se comparados com transações imobiliárias.

\section{METODOLOGIA DA PESQUISA}

\section{CARACTERIZAÇÃO DA PESQUISA}

O método de pesquisa empregado neste trabalho refere-se ao Dedutivo, que é definido por Lakatos e Marconi (1991) como àquele na qual a busca por soluções parte de teorias ou leis previamente aceitas. No que se refere a natureza da aplicação o trabalho pode ser caracterizado como uma Pesquisa Aplicada, pois utiliza-se de conhecimentos provenientes de pesquisas básicas bem como as tecnológicas existentes, visando promover a geração de novos produtos e processos.

No que diz respeito aos objetivos da pesquisa, esse trabalho pode ser caracterizado como uma Pesquisa Descritiva, pois segundo Gil (2002), a pesquisa descritiva estabelece relações entre variáveis por meio da descrição das características de determinado fenômeno ou população.

Essa pesquisa ainda pode ser caracterizada levando em conta à aquisição de referências bibliográficas. Nesse sentido, o referido trabalho pode ser visto como uma Pesquisa Bibliográfica que objetiva conhecer as principais contribuições sobre os fundos estudados.

A pesquisa pode ainda ser caracterizada em função do tempo de coleta e aplicação (dimensão do tempo). Nesse caso, o referido trabalho pode ser visto como um Estudo Longitudinal uma vez que os dados coletados descrevem tendências e eventos ao longo do tempo (Hair Jr. et al., 2005).

Em relação aos Procedimentos Técnicos utilizados esse trabalho de pesquisa pode ser classificado como uma Pesquisa com Delineamento ex-post facto. Segundo Gil (2002) a classificação desse trabalho se enquadra com esse tipo de pesquisa, pois se refere aos experimentos realizados após a ocorrência dos fatos e, sendo assim, o pesquisador não tem controle sobre as variáveis analisadas. 


\section{COLETA DOS DADOS}

Os dados são secundários disponibilizados pelos FII e CVM no formato de Relatório da Instituição Administradora para o exercício findo em 31 de Dezembro de 2007, composto de Relatório da Administração, Balanços Patrimoniais, Demonstração de Resultados, Demonstração dos Fluxos de Caixa, e Parecer dos Auditores Independentes. Foram incorporados à base de dados decorrentes dos Relatórios, EVA - Economic Value Added, ou Valor Econômico Adicionado e EBITDA - Earnings Before Interest, Taxes, Depreciation and Amortization ou Lucro Antes dos Impostos, Depreciação e Amortização como indicadores econômico e financeiro, respectivamente, e ROA - Return on Assets ou Retorno sobre os Ativos, e ROE - Return on Equity ou Retorno sobre Patrimônio Líquido como indicadores de rentabilidade.

\section{MÉTODO DE ANÁLISE: DATA ENVELOPMENT ANALYSIS}

A métrica $D E A$ é uma aplicação da técnica de programação linear utilizada para medir a eficiência relativa de uma determinada unidade operativa em relação às demais unidades com alguns objetivos definidos. Os gestores têm interesse em determinar quão a sua unidade é eficiente em relação às demais unidades da organização ou do mercado utilizando recursos semelhantes e objetivos obtidos. A $D E A$ determina quão é eficiente uma determinada unidade operativa em relação às demais convertendo os recursos de entrada e de saída comparáveis.

Usando a métrica $D E A$, a eficiência de uma determinada unidade é definida por meio da expressão(1).

$$
\text { Eficiência }=\frac{\text { Peso Somatório Output }}{\text { Peso Somatório Input }}=\frac{\sum_{j=1}^{n_{0}} O_{i j} W_{j}}{\sum_{j=1}^{n_{1}} I_{i j} V_{j}}
$$

Onde, “Oij” representa o valor da unidade "i" do output, que é a variável de saída, "j”. "Iij” representa o valor da unidade "i" do input, refere-se a variável de entrada, "j". "Wij" é o peso calculado e atribuído para output "j" e "Vj" é o peso calculado e atribuído para input "j". "n0" é o número de variáveis de saída, output e "ni" o número de variáveis de saída, input.

O problema é resolvido por meio da técnica de programação linear definindo maximização do somatório de saídas com os correspondentes pesos, tal como encontra-se em (2).

$$
\text { Maximizar : } \sum_{\mathrm{j}=1}^{\mathrm{n}_{0}} \mathrm{O}_{\mathrm{ij}} \mathrm{W}_{\mathrm{j}}
$$

Onde a métrica $D E A$ calcula a eficiência para cada unidade em relação às demais. Na $D E A$, em função da programação linear, definem-se as restrições tal como encontra-se descrito em (3) e (4), respectivamente.

$$
\begin{aligned}
& \text { Inequação: } \sum_{\mathrm{j}=1}^{\mathrm{n}_{0}} \mathrm{O}_{\mathrm{ij}} \mathrm{W}_{\mathrm{j}} \leq \sum_{\mathrm{j}=1}^{\mathrm{n}_{1}} \mathrm{I}_{\mathrm{ij}} \mathrm{V}_{\mathrm{j}} ; \text { para } \mathrm{k}=1,2,3, \ldots \text { até o número de unidades. } \\
& \text { Igualdade: } \sum_{\mathrm{j}=1}^{\mathrm{n}_{1}} \mathrm{I}_{\mathrm{ij}} \mathrm{V}_{\mathrm{j}}=1
\end{aligned}
$$

A definição das variáveis input e output depende do problema de eficiência a ser analisado. Vale salientar que as fronteiras de eficiência criadas pelo modelo são empregadas para avaliar a eficiência relativa dos planos de operação executados pelas DMU's (servindo também como referência para o estabelecimento de metas eficientes para cada uma delas. A principal finalidade da Análise por Envoltória de Dados (DEA) é, portanto, a de estimar a as eficiências dos planos de operaçãa executados 
por unidades produtivas homogêneas, que usam um mesmo conjunto de recursos para produzir um mesmo conjunto de resultados, por meio de processos tecnológicos similares (Belloni, 2000; Macedo, 2004; Pereira, 1995; Mello et al., 2004).

\section{APRESENTAÇÃO E ANÁLISE DOS RESULTADOS}

\section{OS FUNDOS DE INVESTIMENTOS IMOBILIÁRIOS}

Os Fundos de Investimentos Imobiliários considerados neste estudo estão registrados na CVM em 31 de Maio de 2008. São 72 Fundos totalizando R\$ 3,47 bilhões em patrimônio líquido. Os dados, compilados das demonstrações financeiras publicadas em 31 de Dezembro de 2007, com dados de 2006, também, servem de base para definir as variáveis de entrada e de saída, input e output, respectivamente. Foram calculados os seguintes valores e indicadores adicionais:

a) EVA - Economic Value Added (Valor Econômico Adicionado):

- EVA 2007 = (Lucro Líquido 2007 - Patrimônio Líquido 2006) x TJLP médio 2007 Onde, a Taxa de Juros de Longo Prazo (TJLP) refere-se ao valor médio no ano de 2007 é 6,375\%, de acordo com Banco Central do Brasil.

b) EBITDA - Earnings Before Interest, Taxes, Depreciation and Amortization (Lucro Antes dos Impostos, Depreciação e Amortização):

- EBITDA = Lucro Líquido + Depreciação - Receita de Aplicações Financeiras

c) ROA - Return on Assets (Retorno sobre os Ativos):

- ROA = (Lucro Líqudo / Ativo Total 2006) x 100

d) ROE - Return on Equity, (Retorno sobre Patrimônio Líquido):

- ROE = (EVA / Patrimônio Líquido 2006) x 100

Os indicadores supracitados contemplam o retorno sobre valores que representam saldo inicial do exercício, ou seja, valores de Ativo Total e Patrimônio Líquido de 31 de Dezembro de 2006. Os FII considerados no estudo foram selecionados de acordo com os seguintes critérios:

a) 0 Fundo realizou operações em 2007;

b) Os dados das Demonstrações Financeiras referem-se aos anos de 2006 e 2007;

c) Legibilidade das Demonstrações Financeiras;

d) Excluem-se os Fundos identificados nos itens a, b e c, que representem, no mínimo, 70\% do patrimônio líquido total.

Assim, 17 Fundos foram selecionados para a pesquisa, o que representa aproximadamente $76 \%$ do patrimônio líquido total, tal como encontra-se evidenciado no Quadro 1: 
Quadro 1: Fundos de Investimentos Imobiliários

\begin{tabular}{|c|c|c|c|}
\hline \multirow{2}{*}{\multicolumn{2}{|c|}{ RAZÃO SOCLAL DO FUNDO }} & \multicolumn{2}{|c|}{ Patrimônio Líquido R\$ Mil } \\
\hline & & $31 / \mathrm{mai} / 08$ & $31 / \mathrm{dez} / 07$ \\
\hline 1 & FII TORRE NORTE & 402.395 & 299.296 \\
\hline 2 & FII PANAMBY & 295.260 & 287.705 \\
\hline 3 & FII SHOPPING PARQUE D PEDRO & 182.564 & 186.287 \\
\hline 4 & OPPORTUNITY FII & 128.181 & 78.260 \\
\hline 5 & LAGRA FII & 126.893 & 115.424 \\
\hline 6 & BB FII PROGRESSIVO & 125.087 & 125.642 \\
\hline 7 & FII SHOPPING PATIO HIGIENOPOLIS & 95.615 & 95.976 \\
\hline 8 & MINAS INDUSTRIAL FII & 95.450 & 95.821 \\
\hline 9 & FII TORRE ALMIRANTEE & 90.874 & 92.349 \\
\hline 10 & FII PROJ AGUA BRANCA & 81.553 & 82.174 \\
\hline 11 & FII ED ALMIRANTE BARROSO & 81.380 & 82.938 \\
\hline 12 & FII VIA PARQUE SHOPPING & 80.866 & 80.226 \\
\hline 13 & FII FATOR-RIO OFFICE PARK & 76.169 & 85.522 \\
\hline 14 & FII PATEO MOINHOS DE VENTO & 62.337 & 62.336 \\
\hline 15 & FII CONTINENTAL SQUARE FARIA LIMA & 60.651 & 61.667 \\
\hline 16 & HG BRASIL SHOPPING FII & 59.672 & 60.286 \\
\hline 17 & FII NOSSA SENHORA DE LOURDES & 55.290 & 56.106 \\
\hline
\end{tabular}

Os Fundos são analisados sob o contexto da principal atividade operacional, venda ou locação; alocação do recurso em imóveis como shopping, residencial, comercial, industrial, etc; e localização dos imóveis, conforme demonstra o Quadro 2.

Quadro 2: Contexto Operacional dos FII

\begin{tabular}{|c|c|c|c|c|}
\hline & RAZÃO SOCIAL DO FUNDO & Imóvel & Renda & Localização \\
\hline 1 & FII TORRE NORTE & Comercial & Locação & São Paulo \\
\hline 2 & FII PANAMBY & Residencial & Venda & São Paulo \\
\hline 3 & FII SHOPPING PARQUE D PEDRO & Shopping & Locação & Campinas \\
\hline 4 & OPPORTUNITY FII & Residencial & Venda & Rio de Janeiro \\
\hline 5 & LAGRA FII & Comercial & $\begin{array}{c}\text { Venda } \\
\text { Locação }\end{array}$ & Rio de Janeiro \\
\hline 6 & BB FII PROGRESSIVO & Comercial & Locação & $\begin{array}{c}\text { Brasília } \\
\text { Rio de Janeiro }\end{array}$ \\
\hline 7 & FII SHOPPING PATIO HIGIENOPOLIS & Shopping & Locação & São Paulo \\
\hline 8 & MINAS INDUSTRIAL FII & Industrial & Locação & Betim \\
\hline 9 & FII TORRE ALMIRANTE & Comercial & Locação & Rio de Janeiro \\
\hline 10 & FII PROJ AGUA BRANCA & Comercial & Locação & São Paulo \\
\hline 11 & FII ED ALMIRANTE BARROSO & Comercial & Locação & Rio de Janeiro \\
\hline 12 & FII VIA PARQUE SHOPPING & Shopping & Locação & Rio de Janeiro \\
\hline 13 & FII FATOR-RIO OFFICE PARK & Comercial & Locação & Rio de Janeiro \\
\hline 14 & FII PATEO MOINHOS DE VENTO & Shopping e Hotel & Locação & Porto Alegre \\
\hline 15 & FII CONTINENTAL SQUARE FARIA LIMA & Residencial & Locação & São Paulo \\
\hline 16 & HG BRASIL SHOPPING FII & Shopping & Locação & São Paulo \\
\hline 17 & FII NOSSA SENHORA DE LOURDES & Hospital & Locação & São Paulo \\
\hline
\end{tabular}

Vale destacar que dos 17 Fundos, 14 atuam em São Paulo e Rio de Janeiro; apenas 3 atuam com vendas e as demais com locação; 7 com imóvel comercial, 5 com shopping, 2 residencial, e 1 hospital, hotel, industrial.

\section{AVALIAÇÃO DA EFICIÊNCIA DOS FUNDOS DEINVESTIMENTOS IMOBILIÁRIOS}

Na definição das variáveis, bem como dos objetivos estabelecidos por meio da métrica $D E A$, são considerados dois estágios:

a) Estágio 1:

- Input: Patrimônio Líquido (R Mil)

Ativo Total (R\$ Mil)

- Output: Receita (R\$ Mil) 
b) Estágio 2:

- Input: Receita (R\$ Mil)

- Output: EVA (R\$ Mil)

EBITDA (R\$ Mil)

c) Estágio Único:

- Input: Patrimônio Líquido (R Mil)

Ativo Total (R\$ Mil)

- Output: ROE (\%)

ROA (\%)

O estudo da eficiência em 3 estágios tem o objetivo de analisar a eficiência dos Fundos no contexto operacional a partir do Ativo Total e Patrimônio Líquido, além da Receita; ou seja, da estrutura de capital e da geração de recursos, respectivamente. A Figura 1 demonstra esquematicamente os estágios analisados, com os seus respectivos input e output.

Figura 1: Modelo de Avaliação em 2 Estágios e de EstágioÚnico

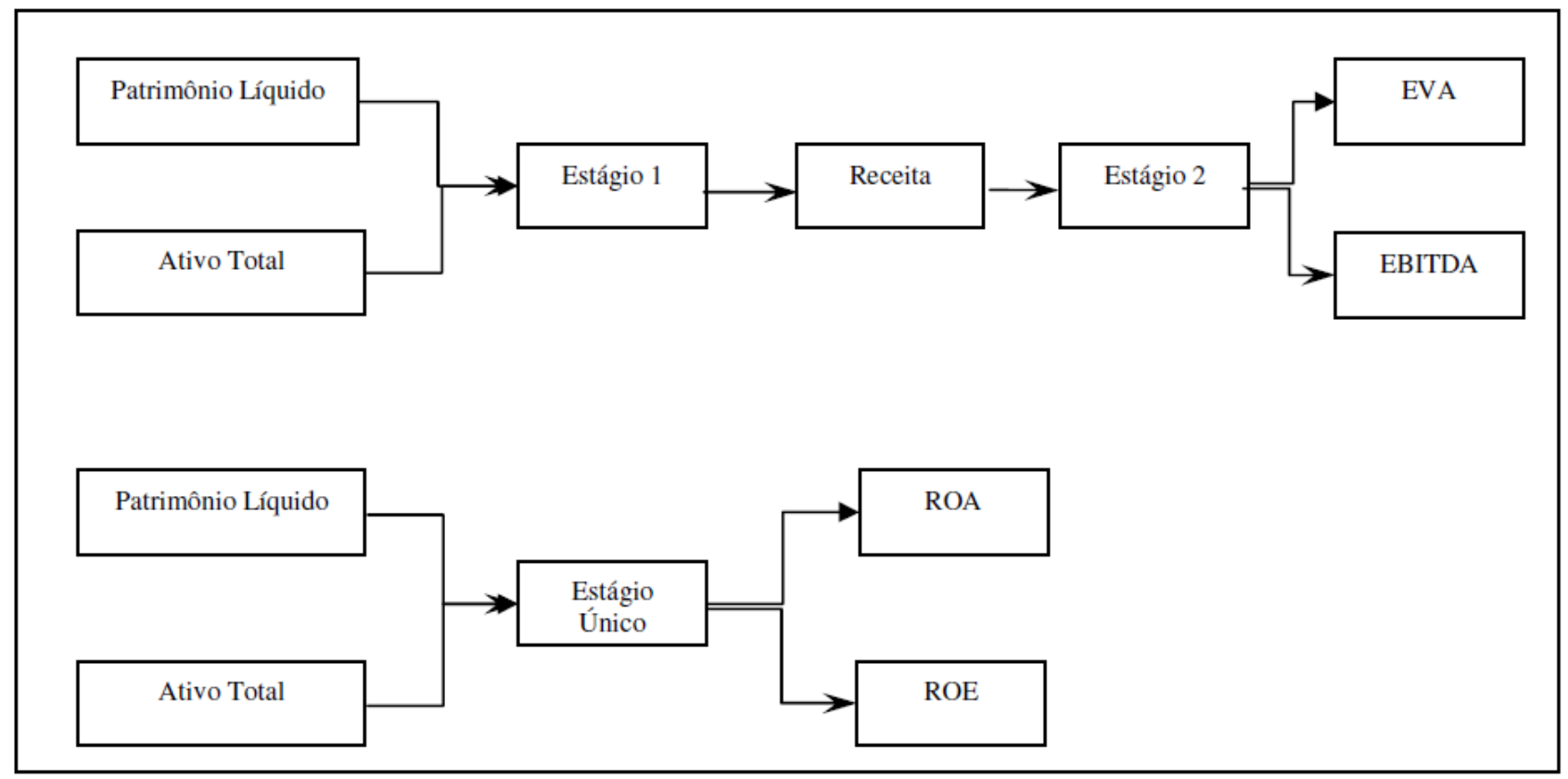

Os dados utilizados nesta pesquisa encontram-se demonstrados nas Tabelas 1, 2 e 3, referentes aos Estágios 1, 2 e Único, respectivamente. 
Tabela 1: Dados Utilizados no Estudo - Estágio 1 - Valores em R \$ Mil

\begin{tabular}{llrrr}
\hline & & \multicolumn{1}{c}{ Output } & \multicolumn{1}{c}{ Input } & Input \\
\cline { 3 - 5 } & & Receita & Ativo & PL \\
\cline { 3 - 5 } 1 & FII TORRE NORTE & 2.007 & 2.007 & 2.007 \\
\hline 2 & FII PANAMBY & 44.692 & 304.098 & 299.296 \\
3 & FII SHOPPING PARQUE D PEDRO & 38.450 & 292.825 & 287.705 \\
4 & OPPORTUNITY FII & 52.362 & 189.366 & 186.287 \\
5 & LAGRA FII & 38.896 & 117.698 & 78.260 \\
6 & BB FII PROGRESSIVO & 43.854 & 168.199 & 115.424 \\
7 & FII SHOPPING PATIO HIGIENOPOLIS & 19.840 & 127.870 & 125.642 \\
8 & MINAS INDUSTRIAL FII & 10.998 & 96.917 & 95.976 \\
9 & FII TORRE ALMIRANTE & 9.869 & 96.135 & 95.821 \\
10 & FII PROJ AGUA BRANCA & 14.080 & 93.604 & 92.349 \\
11 & FII ED ALMIRANTE BARROSO & 19.509 & 84.173 & 82.174 \\
12 & FII VIA PARQUE SHOPPING & 18.386 & 84.507 & 82.938 \\
13 & FII FATOR-RIO OFFICE PARK & 20.463 & 80.479 & 80.226 \\
14 & FII PATEO MOINHOS DE VENTO & 9.341 & 86.432 & 85.522 \\
15 & FII CONTINENTAL SQUARE FARIA LIMA & 13.626 & 65.089 & 62.336 \\
16 & HG BRASIL SHOPPING FII & 5.414 & 62.172 & 61.667 \\
17 & FII NOSSA SENHORA DE LOURDES & 5.546 & 60.413 & 60.286 \\
\hline
\end{tabular}

Os resultados obtidos para a análise da eficiência por meio da métrica $D E A$ dos FII são apresentados na Tabela 3, ordenado pelo principal contexto operacional, da origem da renda, por tipo de imóvel. Os dados utilizados demonstrados nas Tabelas 1, 2 e 3, respectivamente, foram processados por meio da planilha eletrônica Excel 2003®, além do suplemento de otimização Solver, definindo-se o objetivo, as restrições e variáveis representando os pesos. Foram 17 resoluções para cada estágio, totalizando 51 problemas resolvidos.

Tabela 2: Dados Utilizados na Pesquisa - Estágio 2 - Valores em R \$ Mil

\begin{tabular}{|c|c|c|c|c|}
\hline \multirow{2}{*}{\multicolumn{2}{|c|}{ RAZÃO SOCIAL DO FUNDO }} & $\begin{array}{c}\text { Output } \\
\text { EBITDA }\end{array}$ & $\frac{\text { Output }}{\text { EVA }}$ & $\begin{array}{c}\text { Input } \\
\text { Receita }\end{array}$ \\
\hline & & 2.007 & 2.007 & 2.007 \\
\hline 1 & FII TORRE NORTE & 40.942 & 5.977 & 44.692 \\
\hline 2 & FII PANAMBY & 28.540 & 10.535 & 38.450 \\
\hline 3 & FII SHOPPING PARQUE D PEDRO & 46.667 & 27.985 & 52.362 \\
\hline 4 & OPPORTUNITY FII & 7.332 & 2.259 & 38.896 \\
\hline 5 & LAGRA FII & 31.482 & 21.375 & 43.854 \\
\hline 6 & BB FII PROGRESSIVO & 18.793 & 8.786 & 19.840 \\
\hline 7 & FII SHOPPING PATIO HIGIENOPOLIS & 9.236 & 4.898 & 10.998 \\
\hline 8 & MINAS INDUSTRIAL FII & 8.362 & 721 & 9.869 \\
\hline 9 & FII TORRE ALMIRANTE & 13.118 & 2.789 & 14.080 \\
\hline 10 & FII PROJ AGUA BRANCA & 14.578 & 7.497 & 19.509 \\
\hline 11 & FII ED ALMIRANTE BARROSO & 17.230 & 8.096 & 18.386 \\
\hline 12 & FII VIA PARQUE SHOPPING & 36.750 & 32.821 & 20.463 \\
\hline 13 & FII FATOR-RIO OFFICE PARK & 4.399 & 277 & 9.341 \\
\hline 14 & FII PATEO MOINHOS DE VENTO & 8.137 & 3.047 & 13.626 \\
\hline 15 & FII CONTINENTAL SQUARE FARIA LIMA & 4.763 & $(2.174)$ & 5.414 \\
\hline 16 & HG BRASIL SHOPPING FII & 4.276 & 2.283 & 5.546 \\
\hline 17 & FII NOSSA SENHORA DE LOURDES & 9.331 & 3.272 & 9.669 \\
\hline
\end{tabular}


Tabela 3: Dados Utilizados no Estudo - Estágio Único - Valores em R M Mil

\begin{tabular}{|c|c|c|c|c|c|}
\hline & \multirow{3}{*}{ RAZÃO SOCIAL DO FUNDO } & \multirow{3}{*}{$\begin{array}{c}\text { Output } \\
\text { ROA } \\
2.007\end{array}$} & \multirow{3}{*}{$\begin{array}{c}\text { Output } \\
\text { ROE } \\
2.007\end{array}$} & \multirow{3}{*}{$\begin{array}{l}\text { Input } \\
\text { Ativo } \\
2.007\end{array}$} & \multirow{3}{*}{$\begin{array}{c}\text { Input } \\
\text { PL } \\
2.007\end{array}$} \\
\hline & & & & & \\
\hline & & & & & \\
\hline 1 & FII TORRE NORTE & 0,0814 & 0,0190 & 304.098 & 299.296 \\
\hline 2 & FII PANAMBY & 0,0994 & 0,0373 & 292.825 & 287.705 \\
\hline 3 & FII SHOPPING PARQUE D PEDRO & 0,2077 & 0,1466 & 189.366 & 186.287 \\
\hline 4 & OPPORTUNITY FII & 0,0524 & 0,0556 & 117.698 & 78.260 \\
\hline 5 & LAGRA FII & 0,1667 & 0,1944 & 168.199 & 115.424 \\
\hline 6 & BB FII PROGRESSIVO & 0,1303 & 0,0692 & 127.870 & 125.642 \\
\hline 7 & FII SHOPPING PATIO HIGIENOPOLIS & 0,1298 & 0,0675 & 96.917 & 95.976 \\
\hline 8 & MINAS INDUSTRIAL FII & 0,0698 & 0,0075 & 96.135 & 95.821 \\
\hline 9 & FII TORRE ALMIRANTE & 0,0915 & 0,0289 & 93.604 & 92.349 \\
\hline 10 & FII PROJ AGUA BRANCA & 0,1498 & 0,0899 & 84.173 & 82.174 \\
\hline 11 & FII ED ALMIRANTE BARROSO & 0,1545 & 0,0934 & 84.507 & 82.938 \\
\hline 12 & FII VIA PARQUE SHOPPING & 0,7041 & 0,6433 & 80.479 & 80.226 \\
\hline 13 & FII FATOR-RIO OFFICE PARK & 0,0656 & 0,0032 & 86.432 & 85.522 \\
\hline 14 & FII PATEO MOINHOS DE VENTO & 0,1084 & 0,0481 & 65.089 & 62.336 \\
\hline 15 & FII CONTINENTAL SQUARE FARIA LIMA & 0,0297 & $(0,0339)$ & 62.172 & 61.667 \\
\hline 16 & HG BRASIL SHOPPING FII & 0,1133 & 0,0505 & 60.413 & 60.286 \\
\hline 17 & FII NOSSA SENHORA DE LOURDES & 0,1167 & 0,0562 & 57.666 & 56.106 \\
\hline
\end{tabular}

A eficiência por meio da métrica $D E A$ indica que, quanto mais próximo de 1 estiver o índice de eficiência do fundo avaliado, mais eficiente também será o FII. Observe que o FII Via Parque Shopping é o único que sinalizou eficiência em todos os estágios, que é o décimo segundo colocado em termos de patrimônio líquido.

Os Fundos Opportunity, HG Brasil Shopping, e Continental Square Faria Lima sinalizaram eficiência em pelo menos dois estágios. E, a Lagra, Pateo Moinhos de Vento, Shopping Parque D. Pedro, Minas Industrial, e Nossa Senhora de Lourdes sinalizaram eficiência em um dos estágios. Assim, 8 dos 17 Fundossinalizaram eficiência em pelo menos um estágio, conforme evidencia a Tabela 4.

Tabela 4 - Eficiência DEA dos FII por Renda; Imóvel e Estágio

\begin{tabular}{|c|c|c|c|c|c|}
\hline Renda & Imóvel & RAZÃO SOCIAL DO FUNDO & Estágio 1 & Estágio 2 & $\begin{array}{l}\text { Estágio } \\
\text { Único }\end{array}$ \\
\hline \multirow{14}{*}{ 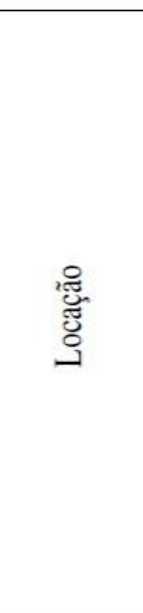 } & \multirow{6}{*}{ Comercial } & BB FII PROGRESSIVO & 0,56 & 0,70 & 0,46 \\
\hline & & FII ED ALMIRANTE BARROSO & 0,79 & 0,69 & 0,71 \\
\hline & & FII FATOR-RIO OFFICE PARK & 0,41 & 0,43 & 0,70 \\
\hline & & FII PROJ AGUA BRANCA & 0,83 & 0,54 & 0,69 \\
\hline & & FII TORRE ALMIRANTE & 0,55 & 0,79 & 0,64 \\
\hline & & FII TORRE NORTE & 0,53 & 0,80 & 0,20 \\
\hline & Hospital & FII N SENHORA DE LOURDES & 0,60 & 0,76 & 1,00 \\
\hline & Industrial & MINAS INDUSTRIAL FII & 0,40 & 0,77 & 1,00 \\
\hline & Residencial & $\begin{array}{c}\text { FII CONTINENTAL SQUARE } \\
\text { FARIA LIMA }\end{array}$ & 0,33 & 1,00 & 1,00 \\
\hline & \multirow{4}{*}{ Shopping } & FII SHOPPING P D PEDRO & 1,00 & 0,61 & 0,33 \\
\hline & & $\begin{array}{c}\text { FII SHOPPING PATIO } \\
\text { HIGIENOPOLIS }\end{array}$ & 0,43 & 0,60 & 0,62 \\
\hline & & FII VIA PARQUE SHOPPING & 1,00 & 1,00 & 1,00 \\
\hline & & HG BRASIL SHOPPING FII & 1,00 & 0,55 & 1,00 \\
\hline & Shopping Hotel & FII PATEO MOINHOS DE VENTO & 0,75 & 0,47 & 0,94 \\
\hline \multirow[b]{2}{*}{$\frac{\pi}{\frac{3}{5}}$} & \multirow[b]{2}{*}{ Residencial } & FII PANAMBY & 0,47 & 0,58 & 0,20 \\
\hline & & OPPORTUNITY FII & 1,00 & 0,15 & 1,00 \\
\hline $\begin{array}{l}\text { Venda e } \\
\text { Locação }\end{array}$ & Comercial & LAGRA FII & 0,80 & 0,47 & 1,00 \\
\hline
\end{tabular}

Vale destacar que os cinco maiores Fundos em patrimônio líquido, a saber: Torre Norte, Panamby, Shopping Parque D.Pedro, Opportunity, e Lagra, invariavelmente sinalizaram eficiência, exceto Panamby, com empreendimentos em contas a receber em função da venda de imóveis.

Ao considerar a eficiência a partir do valor igual a 0,70, ou seja, 70\% de eficiência, somente os Fundos 
Shopping Pátio Higienópolis e Panamby ficam abaixo desse patamar. No caso do Panamby, sugere-se que praticamente não houve operação por conta dos empreendimentos estarem em contas a receber por venda de imóveis. Assim, praticamente todos os Fundos sinalizaram eficiência em pelo menos um estágio.

Ao analisar o principal contexto operacional, conforme o tipo de imóvel, os Fundos que operam com imóveis comerciais têm apresentado eficiência inferior àqueles que operam com Shopping Centers. Sugere-se que a alocação de recurso nesse contexto operacional responde melhor que comercial.

A Tabela 5 demonstra os resultados da $D E A$ eficiência ordenado por patrimônio líquido, de maior para menor. Ao analisar o resultado no Estágio 1, dos 4 Fundos que apresentaram eficiência igual a unidade, 3 operam com shopping center.

Ao analisar o Estágio 2, apenas 2 Fundos atingiram eficiência máxima. E, no estágio único, 7 Fundos atingiram eficiência máxima e um atingiu 0,94 , ou seja, praticamente a metade deles atingiram a eficiência máxima. Sugere-se ainda que ao analisar os estágios com input patrimônio líquido e ativo total, há uma maior eficiência. Enquanto que ao analisar com input receita, a eficiência máxima não é sinalizada com a mesma freqüência. Assim, sugere-se que o montante do patrimônio líquido do Fundo não é determinante para identificar a eficiência.

Os resultados das análises dos Fundos nos 3 estágios com objetivo de maximizar o output a partir da métrica $D E A$ podem ser complementares à análise investimentos convencional por meio de indicadores de rentabilidade, incorporando o enfoque de uso de recursos de forma de forma eficiente.

Tabela 5: Eficiência DEA dos FII Ordenado por Patrimônio Líquido

\begin{tabular}{clccc}
\hline & \multicolumn{2}{c}{ RAZÃO SOCIAL DO FUNDO } & \multicolumn{3}{c}{ DEA Eficiência } \\
\cline { 3 - 5 } & & Estágio 1 & Estágio 2 & Estágio Único \\
\hline 1 & FII TORRE NORTE & 0,53 & 0,80 & 0,20 \\
2 & FII PANAMBY & 0,47 & 0,58 & 0,20 \\
3 & FII SHOPPING PARQUE D PEDRO & 1,00 & 0,61 & 0,33 \\
4 & OPPORTUNITY FII & 1,00 & 0,15 & 1,00 \\
5 & LAGRA FII & 0,80 & 0,47 & 1,00 \\
6 & BB FII PROGRESSIVO & 0,56 & 0,70 & 0,46 \\
7 & FII SHOPPING PATIO HIGIENOPOLIS & 0,43 & 0,60 & 0,62 \\
8 & MINAS INDUSTRIAL FII & 0,40 & 0,77 & 1,00 \\
9 & FII TORRE ALMIRANTE & 0,55 & 0,79 & 0,64 \\
10 & FII PROJ AGUA BRANCA & 0,83 & 0,54 & 0,69 \\
11 & FII ED ALMIRANTE BARROSO & 0,79 & 0,69 & 0,71 \\
12 & FII VIA PARQUE SHOPPING & 1,00 & 1,00 & 1,00 \\
13 & FII FATOR-RIO OFFICE PARK & 0,41 & 0,43 & 0,70 \\
14 & FII PATEO MOINHOS DE VENTO & 0,33 & 0,47 & 0,94 \\
15 & FII CONTINENTAL SQUARE FARIA LIMA & 1,00 & 0,55 & 1,00 \\
16 & HG BRASIL SHOPPING FII & 0,60 & 0,76 & 1,00 \\
17 & FII NOSSA SENHORA DE LOURDES & & 1,00 \\
\hline
\end{tabular}

Os resultados sugerem que os Fundos têm apresentado esforços na eficiência quanto ao uso dos recursos disponíveis. É um sinalizador ao mercado que os Fundos são eficientes, ou seja, sugere eficiência na alocação dos recursos Patrimônio Líquido, Ativo Total e Receita considerando Receita, EVA, EBITDA, ROE e ROA como output.

\section{CONSIDERAÇÕES FINAIS E RECOMENDAÇÕES}

A métrica $D E A$ é uma ferramenta interessante que pode ser incorporada na rotina de análise de performance dos Fundos de Investimentos Imobiliários. É uma métrica que utiliza modelagem do problema de programação linear exigindo pouca preparação e padronização dos dados demonstrando flexibilidade para realizar simulações de resultados.

Ao analisar os resultados obtidos, constata-se que a métrica $D E A$ permite a análise de um determinado Fundo em conjunto com os demais, ou seja, no segmento no qual está inserido. Os resultados deste 
estudo sugerem os Fundos são mais eficientes no uso de recursos Patrimônio Líquido e Ativo Total do que o uso de Receitas. Quando incorpora as características do contexto operativo dos Fundos aos resultados da análise $D E A$, os Fundos com contexto em locação e tipo de imóvel comercial e shopping é predominante, situados em grande parte em São Paulo e Rio de Janeiro.

O presente estudo contemplou 17 Fundos de um total de 72 Fundos registrados na CVM que considerados critérios de seleção, representaram 76\% do total em Patrimônio Líquido. Dentre esses Fundos, os resultados não sugerem que o montante do Patrimônio Líquido seja um dos fatores que determina maior ou menor eficiência.

Os Fundos de Investimentos Imobiliários foram regulamentados em 1993, ou seja, há 15 anos, que pode ser considerado como uma limitação da carência de dados históricos suficientes para realização de pesquisas e identificação de indicadores de performance com o mesmo perfil dos demais Fundos de Investimentos no mercado nacional ou internacional. Embora a eficiência dos FII sejam influenciada pela Receita, como input, EVA e EBITDA, como output, apenas 2 Fundos dentre 17 atingiram eficiência máxima. Assim, é importante que, em futuros trabalhos, possam ampliar o estudo utilizando outros recursos como input e output com objetivo de incorporar novos subsídios para análise da performance dos Fundos de Investimentos Imobiliários.

0 mercado dos Fundos de Investimentos Imobiliários - FII tem representado uma opção a mais no mercado financeiro para carrear recursos para o financiamento de ativos reais no Brasil. Os resultados econômicos e financeiros relativo aos anos 2006 e 2007 demonstraram que mesmo atuando em segmentos similares os Fundos de Investimentos fundos imobiliários apresentaram indicadores de performance operacional com grandes diferenças.

Percebeu-se que a teoria Visão Baseada em Recursos apresentou aderência aos aspectos que explicam as razões de forma abrangente, da ocorrência de grandes diferenças de desempenho dada a existência de vantagem competitiva nos Fundos de Investimentos Imobiliários analisados. Além da heterogeneidade dos recursos utilizados pelos Fundos, existem os efeitos decorrentes das idiossincrasias reconhecidas por Rumelt (1991) e McGahan (1997) como importantes na explicação das diferenças dos resultados das empresas de um mesmo setor.

\section{REFERÊNCIAS}

ANDERSON, D. R. An Introduction to Management Science: Quantitative Approaches to Decision Making. Thoson South: Western Publishing, 2005.

ANDREWS, K. R.; The concept of corporate strategy. In: McKierman, Peter (ed.). Historical Evolution of Strategic Management. Brookfield: Darmouth Publishing Company, 1980 p.15-44.

BARBOSA, S. de L.; SILVA, W. V. da; SILVA, E. D.; CORSO, J. M. D. Avaliação da Eficiência Docente em um Programa de Pós-Graduação Stricto Sensu na Região Sul do Brasil com o uso de Data Envelopment Analysis - DEA XXXI EnANPAD, Rio de Janeiro - RJ, 22 a 26 de setembro de 2007.

BARNEY, J. Firm resources and sustained competitive advantage, Journal of Management. v. 17, n. 99-120, 1991.

BECKER, J. L.; LUNARDI, G. L.; MAÇADA, A. C. G. Análise de Eficiência dos Bancos Brasileiros: um enfoque nos Investimentos Realizados em Tecnologia da Informação (TI). Revista Produção, v. 13 n. 2 2003

BOVESPA, Bolsa de Valores de São Paulo. Brasil Disponível em: <http://www.bovespa.com.br/> Acesso em: 09 a 27/06/2008.

CVM, Comissão de Valores Mobiliários Brasil. Disponível em: http://www.cvm.gov.br/Acesso em: 09 a $27 / 06 / 2008$.

GIL, A. C. Como elaborar projetos de pesquisa. 4aㅡ ed. São Paulo: Atlas, 2002. 
GONÇALVES, D. A.; LINS, M. P. E. Análise Envoltória de Dados (DEA) na Avaliação de Eficiência de Fundos de Investimentos. XXXII Simpósio Brasileiro de Pesquisa Operacional. Viçosa, 18 a 20 de Outobro de 2000.

GONÇALVES, D. A.; MELLO, J. C. C. B. S.; LINS, M. P. E. Classificação de Fundos de Investimentos Usando um Índice de Sharpe Modificado. XXXII Simpósio Brasileiro de Pesquisa Operacional. Viçosa, 18 a 20 de Outubro de 2000.

HAIR, Jr. J. F. et al. Fundamentos de Métodos de Pesquisa em Administração. Porto Alegre: Bookman, 2005.

JOHNSON, G. Explorando a Estratégia Corporativa: Texto e Casos, Gerry Johnson, Kevan Scholes, Richard Whittington; tradução de Luciana de Oliveira da Rocha - 7. ed. - Porto Alegre: Bookman, 2007.

LAKATOS, E. M.; MARCONI, M. A. Fundamentos da Metodologia Científica. São Paulo: Atlas, 1991.

MINTZBERG, H. et al.; O Processo da Estratégia: conceitos, contextos e casos selecionados; Sumantra Ghoshal, Joseph Lampel, Henry Mintzberg, James BrianQuinn; tradução Luciana de Oliveira da Rocha. Porto Alegre: Bookman, 2006.

MUGNAINI, Alexandre. Análise da Eficiência de Mercado e Performance de Fundos de Investimentos Imobiliários Negociados na Bovespa. Dissertação de Mestrado apresentada ao Programa de PósGraduaçãi em Administração PUCPR, Curitiba 2007.

PORTER, M. E. Estratégia Competitiva: técnicas para análise de indústrias e da concorrência. Tradução Elizabeth Maria de Pinho. Rio de Janeiro: Campus, 1989.

SAYAN, C. Estratégias à prova de falhas: como lucrar e crescer correndo riscos que outros evitam. Porto Alegre: Bookman, 2006.

RAGSDALE, C. Managerial Decision Modeling. Cliff Ragsdale. Paperback, I.S. ed. Edition, SouthWestern, Div of Thomson Learning, 2006.

TEECE, D. J., G. Pisano, and A. Schuen. Dynamic capabilities and strategic management, Strategic Management Journal, v. 18, p. 509-533, 1997.

WINSTON, W. L. Practical Management Science. Wayne L. Winston, S. Christian Albright. Duxbury, 1997. 